PROCEEDINGS OF THE

AMERICAN MATHEMATICAL SOCIETY

Volume 137, Number 8, August 2009, Pages 2785-2794

S 0002-9939(09)09916-X

Article electronically published on April 6, 2009

\title{
A SPECTRAL THEORETIC APPROACH TO THE KIRILLOV-DUFLO CORRESPONDENCE
}

\author{
R. W. RAFFOUL
}

(Communicated by Varghese Mathai)

\begin{abstract}
The Kirillov-Duflo orbit correspondance for compact Lie groups is parametrisation of the unitary dual, associating to the irreducible representation of highest weight $\lambda$ the coadjoint orbit through $\lambda+\delta$, where $\delta$ is half the sum of the positive roots and justified by the character formulae of Weyl or Kirillov. In this paper we obtain this correspondence independently of character theory, showing that it arises from a convexity property of the Weyl functional calculus of the infinitesimal generators of the representation.
\end{abstract}

\section{INTRODUCTION}

Let $G$ be a semisimple compact connected Lie group with Lie algebra $\mathfrak{g}$ and let $\mathfrak{g}^{*}$ be the vector space dual of $\mathfrak{g}$. Fix a Cartan subalgebra $\mathfrak{t}$, denote the Weyl group by $W$ and distinguish a positive Weyl chamber. Let $\mathfrak{t}^{r e g}$ denote the set of regular elements of $\mathfrak{t}$, i.e. those $H$ for which $i H$ does not belong to a wall of any Weyl chamber.

Let $\pi$ be an irreducible representation of $G$ with highest weight $\lambda$. Denote by $\delta$ the half-sum of the positive roots of $(\mathfrak{g}, \mathfrak{t})$. The Weyl character formula states that the character $\chi_{\pi}$ is given by the closed-form expression

$$
\chi_{\pi}(\exp H)=\frac{\sum_{w \in W} \operatorname{sgn}(w) e^{w(\lambda+\delta)(H)}}{\Delta(H)}
$$

for all $H \in \mathfrak{t}^{r e g}$, where $\Delta$ is the Weyl denominator function defined as

$$
\Delta(H):=\sum_{w \in W} \operatorname{sgn}(w) e^{w \delta(H)}
$$

for all $H \in \mathfrak{t}$. This relation implies a parametrisation of the set of irreducible representations of $G$ by positive integral weights in $\mathfrak{t}^{*}$, whereby a representation $\pi$ of highest weight $\lambda$ is associated to the linear functional $\lambda+\delta$.

A still more precise form of this correspondence is achieved by the Kirillov character formula. On every coadjoint orbit $\mathcal{O}$ of $G$, the Kirillov-Kostant-Souriau 2-form $\omega_{\mathcal{O}}$ is defined by

$$
\omega_{\mathcal{O}}(\xi)\left(a d^{*}(X) \xi, a d^{*}(Y) \xi\right):=\langle\xi,[X, Y]\rangle
$$

for all $X, Y \in \mathfrak{g}$ and $\xi \in \mathfrak{g}^{*}$, and it endows the manifold $\mathcal{O}$ with a symplectic structure. If $2 d$ is the dimension of the orbit $\mathcal{O}$, then the expression $\frac{1}{d} \omega_{\mathcal{O}} \wedge \cdots \wedge \omega_{\mathcal{O}}$

Received by the editors August 13, 2008.

2000 Mathematics Subject Classification. Primary 54C40, 14E20; Secondary 46E25, 20C20.

Key words and phrases. Lie groups, coadjoint orbits.

(C)2009 American Mathematical Society 2785

Reverts to public domain 28 years from publication 
( $d$ times) is the Liouville measure $\mu_{\mathcal{O}}$ on $\mathcal{O}$. The following relation was first proved by Kirillov [9]:

$$
j(H) \chi_{\pi}(\exp H)=\int_{\mathcal{O}_{\lambda+\delta}} e^{i \beta(H)} d \mu_{\mathcal{O}_{\lambda+\delta}}(\beta),
$$

where $\mathcal{O}_{\lambda+\delta}$ denotes the coadjoint orbit through $\lambda+\delta$ and

$$
j(X):=\operatorname{det}^{1 / 2}\left(\frac{\sinh a d(X / 2)}{a d(X / 2)}\right)
$$

for all $X \in \mathfrak{g}$. Here, a one-to-one correspondence between irreducible representations and coadjoint orbits is explicit. This is known as the "Kirillov-Duflo correspondence" and is applicable to a wide variety of Lie groups (see [13] for a survey).

In the sequel, we derive this correspondence without resorting to character theory 11 To the unitary irreducible representation $\pi$, we associate a vector $d \pi(X)$ of self-adjoint operators given by the values of the derived representation $d \pi$ on a fixed basis of $X$ of $\mathfrak{g}$, orthonormal with respect to the Killing form. It is shown that the set of extremal points of the convex hull of the support of the Weyl functional calculus distribution of $d \pi(X)$ is exactly the orbit $\mathcal{O}_{\lambda}$ (Theorem 6.12). The bijection $\pi \leftrightarrow \mathcal{O}_{\lambda+\delta}$ is obtained by convolving the Weyl calculus of $d \pi(X)$ with the Fourier transform of the function $j$ defined above (Theorem 6.15).

Key to our analysis is a result of E. Nelson which states that the support of the Weyl calculus of a $d$-tuple of hermitian elements in a Banach algebra is equal to the joint spectrum of the same $d$-tuple in a certain commutative Banach algebra of "operants". We present this fact in Section 4 (Theorem 4.9), after some background material is introduced.

The Nelson algebra of operants is a special case of the more general notion of "operating algebras", introduced by E. Albrecht [1], which are discussed in the next section.

The author thanks A. Dooley for many helpful discussions on this work throughout the course of the former's PhD degree, and M. Duflo for providing much useful feedback on an earlier version of this article. The support of the Australian Research Council is also gratefully acknowledged.

\section{Operating Algebras}

Let $\Re$ be a complex unital Banach algebra with unit $1_{\mathfrak{R}}$. Let $E$ be any vector subspace of $\mathfrak{R}$ and let $\mathfrak{D}$ be a complex unital Banach algebra with unit $1_{\mathfrak{D}}$. Let $\phi: E \rightarrow \mathfrak{D}$ be a one-to-one linear mapping and denote by $\langle\phi(E)\rangle$ the subalgebra of $\mathfrak{D}$ generated by $\phi(E)$. Denote by $\mathcal{S}_{n}$ the symmetric group of order $n$.

Definition 2.1 (1). A commutative complex unital Banach algebra $\mathfrak{D}$ is called an operating algebra with respect to $E$ and $\mathfrak{R}$ if there exists a one-to-one linear mapping $\phi: E \rightarrow \mathfrak{D}$ such that $\langle\phi(E)\rangle$ is dense in $\mathfrak{D}$ and a continuous linear mapping $\operatorname{Sym}_{\mathfrak{R}}^{(\mathfrak{D}, \phi)}: \mathfrak{D} \rightarrow \mathfrak{R}$ determined uniquely by the conditions $\operatorname{Sym}_{\mathfrak{R}}^{(\mathfrak{D}, \phi)}\left(1_{\mathfrak{D}}\right)=1_{\mathfrak{R}}$ and

$$
\operatorname{Sym}_{\mathfrak{R}}^{(\mathfrak{D}, \phi)}\left(y_{1} \ldots y_{n}\right):=\frac{1}{n !} \sum_{\sigma \in \mathcal{S}_{n}} \phi^{-1} y_{\sigma(1)} \cdots \phi^{-1} y_{\sigma(n)}
$$

for all $y_{1}, \ldots, y_{n} \in\langle\phi(E)\rangle, n \in \mathbb{N}$.

\footnotetext{
${ }^{1}$ See [7] for an alternative approach using Dirac operators.
} 
Let $S(E)$ be the symmetric algebra of $E$ and let ${ }^{\wedge}: E \rightarrow S(E)$ be the canonical inclusion mapping. It is well-known that any $\alpha \in S(E)$ can be written in the form

$$
\alpha=a+\sum_{i=1}^{n} \hat{x}_{j_{1}}^{(i)} \cdots \hat{x}_{j_{i}}^{(i)}
$$

where $a \in \mathbb{C}, n, j_{1}, \ldots, j_{n} \in \mathbb{N}$ and $x_{k}^{(j)} \in E$.

It can be checked [11] that the formula

$$
\|\alpha\|_{S(E)}:=\inf \left\{|a|+\sum_{i=1}^{n}\left\|x_{j_{1}}^{(i)}\right\|_{\Re} \cdots\left\|x_{j_{i}}^{(i)}\right\|_{\mathfrak{R}}\right\},
$$

where the infimum is taken over all representations of $\alpha$ of the form (2.2), defines an algebra norm on $S(E)$. The completion of $S(E)$ with respect to this norm is a commutative Banach algebra, which we denote $\bar{S}(E)$. For simplicity of notation, we write $\operatorname{Sym}_{\mathfrak{R}}^{(E)}$ for $\operatorname{Sym}_{\mathfrak{R}}^{\left(\bar{S}(E),{ }^{\wedge}\right)}$.

Lemma 2.2. The complete symmetric algebra $\bar{S}(E)$ is an operating algebra with respect to $E$ and $\Re$.

Proposition 2.3 ([1], Proposition 3.3). Every operating algebra $\mathfrak{D}$ with respect to $E$ and $\mathfrak{R}$ is isometrically isomorphic to the completion of a quotient of $S(E)$ endowed with some algebra norm.

\section{NumERICAL RANGES AND HERMITIAN ELEMENTS}

Let $\mathfrak{R}^{*}$ be the dual space of continuous linear functionals on $\mathfrak{R}$. By the HahnBanach theorem the following set is not void:

$$
D(\Re, 1):=\left\{f \in \mathfrak{R}^{*}: f\left(1_{\mathfrak{R}}\right)=1=\|f\|\right\} .
$$

This enables us to make the next

Definition 3.1. Let $x=\left(x_{1}, \ldots, x_{d}\right) \in \mathfrak{R}^{d}$. The numerical range of $x$ with respect to $\mathfrak{R}$ is the set $V_{\mathfrak{R}}(x):=\left\{\left(f\left(x_{1}\right), \ldots, f\left(x_{d}\right)\right) \mid f \in D(\Re, 1)\right\}$. $\Re$.

We note that if $\mathfrak{R}$ is a $C^{*}$-algebra, then $D(\Re, 1)$ is exactly the set of states of

Let $\mathcal{H}$ be a Hilbert space and denote by $L(\mathcal{H})$ the algebra of bounded linear operators on $\mathcal{H}$. It is well-known that the defining representation is the only irreducible representation of $L(\mathcal{H})$; hence, by the GNS construction, the pure states of $L(\mathcal{H})$ are exactly the set of functionals $\phi: L(\mathcal{H}) \rightarrow \mathbb{C}$ of the form $\phi(x):=\langle x u, u\rangle$ $(x \in L(\mathcal{H}))$ for some fixed unit vector $u \in \mathcal{H}$.

Let $A \subseteq \mathbb{C}^{n}$. We write co $A$ for the convex hull of $A$. By the preceding discussion and the KreĬn-Milman theorem we immediately have the following.

Theorem 3.2. Let $\mathfrak{R}=L(\mathcal{H})$ and let $x=\left(x_{1}, \ldots, x_{d}\right) \in \mathfrak{R}^{d}$. Then

$$
V_{L(\mathcal{H})}(x):=\operatorname{co}\left\{\left(\left\langle x_{1} u, u\right\rangle, \ldots,\left\langle x_{d} u, u\right\rangle\right): u \in \mathcal{H},\|u\|=1\right\} .
$$

Lemma 3.3. Let $T: \mathbb{C}^{d} \rightarrow \mathbb{C}^{d}$ be a linear mapping. Let $x=\left(x_{1}, \ldots, x_{d}\right) \in \mathfrak{R}^{d}$. Then $V_{\mathfrak{R}}(T x)=T V_{\mathfrak{R}}(x)$.

Theorem 3.4 ([5], Theorem 1.2.3). Let $x=\left(x_{1}, \ldots, x_{d}\right) \in \mathfrak{R}^{d}$. The numerical range $V_{\mathfrak{R}}(x)$ is a compact, convex subset of $\mathbb{C}^{d}$.

Proof. See [5], Theorem 3, Chapter 1, Section 2. 
An element $x=\left(x_{1}, \ldots, x_{d}\right) \in \mathfrak{R}^{d}$ satisfying the following equivalence will be called hermitian.

Theorem 3.5 ([5], Lemma 2.2.5). Let $x=\left(x_{1}, \ldots, x_{d}\right) \in \mathfrak{R}^{d}$. Then $V_{\mathfrak{R}}(x) \subseteq \mathbb{R}^{d}$ if and only if $\left\|e^{i \xi \cdot x}\right\|=1$ for all $\xi \in \mathbb{R}^{d}$.

Definition 3.6. Let $x=\left(x_{1}, \ldots, x_{d}\right) \in \mathfrak{R}^{d}$. The two sets

$$
\sigma_{\mathfrak{R}}^{L}(x):=\left\{\lambda \in \mathbb{C}^{d}: \sum_{i} \mathfrak{R} \cdot\left(\lambda_{i} 1_{\mathfrak{R}}-x_{i}\right) \neq \mathfrak{R}\right\}
$$

and

$$
\sigma_{\mathfrak{R}}^{R}(x):=\left\{\lambda \in \mathbb{C}^{d}: \sum_{i}\left(\lambda_{i} 1_{\mathfrak{R}}-x_{i}\right) \cdot \mathfrak{R} \neq \mathfrak{R}\right\}
$$

are called respectively the left spectrum and the right spectrum of $x$ with respect to $\mathfrak{R}$. The set $\sigma_{\mathfrak{R}}(x):=\sigma_{\mathfrak{R}}^{L}(x) \cup \sigma_{\mathfrak{R}}^{R}(x)$ is called the spectrum of $x$ with respect to $\mathfrak{R}$.

Theorem 3.7 ([5], Theorem 1.2.12). Let $x=\left(x_{1}, \ldots, x_{d}\right) \in \mathfrak{R}^{d}$. Then $\operatorname{co} \sigma_{\mathfrak{R}}(x) \subseteq$ $V_{\mathfrak{R}}(x)$.

Following [ [5], we denote by $N$ the set of all algebra norms equivalent to the given norm. We write $V_{\mathfrak{R}}^{(p)}(x)$ for the numerical range of $x$ with respect to the norm $p$.

Theorem 3.8 ([5], Theorem 1.2.13). Let $x=\left(x_{1}, \ldots, x_{d}\right) \in \mathfrak{R}^{d}$ be a d-tuple of commuting elements. Then

$$
\operatorname{co} \sigma_{\mathfrak{R}}(x)=\bigcap_{p \in N} V_{\mathfrak{R}}^{(p)}(x)
$$

For the remainder of this section we fix $x=\left(x_{1}, \ldots, x_{d}\right) \in \mathfrak{R}^{d}$. Suppose that $E$ is the linear span of $1_{\mathfrak{R}}$ and $x_{1}, \ldots, x_{d}$, the linear mapping $\phi: E \rightarrow \mathfrak{D}$ satisfies $\|\phi\|=1$, and $\phi\left(1_{\mathfrak{R}}\right)=1_{\mathfrak{D}}$. Let $\phi(x)$ denote the $d$-tuple $\left(\phi\left(x_{1}\right), \ldots, \phi\left(x_{d}\right)\right)$.

Proposition 3.9 ([1], Lemma 5.3). The inclusion

$$
V_{\mathfrak{D}}(\phi(x)) \subseteq V_{\mathfrak{R}}(x)
$$

holds with equality if $\phi$ is an isometry.

Corollary 3.10. Suppose that $\mathfrak{R}$ is a $C^{*}$-algebra and that $x=\left(x_{1}, \ldots, x_{d}\right) \in \mathfrak{R}^{d}$ is a d-tuple of pairwise commuting elements. Then

$$
\operatorname{co} \sigma_{\mathfrak{D}}(\phi(x)) \subseteq V_{\mathfrak{R}}(x)
$$

Proof. By Theorem 3.8 ,

$$
\operatorname{co} \sigma_{\mathfrak{D}}(\phi(x))=\bigcap_{p \in N} V_{\mathfrak{D}}^{(p)}(\phi(x))
$$

and by Lemma 3.9, $V_{\mathfrak{D}}^{(p)}(\phi(x)) \subseteq V_{\mathfrak{R}}^{(p)}(x)$ with equality if $\phi$ is an isometry. Since $\bigcap_{p \in N} V_{\mathfrak{R}}^{(p)}(x) \subseteq V_{\mathfrak{R}}(x)$, the result follows.

Theorem 3.11 (1], Theorem 5.7). Suppose that $\mathfrak{R}$ is a $C^{*}$-algebra, that $x=$ $\left(x_{1}, \ldots, x_{d}\right) \in \mathfrak{R}^{d}$ is a d-tuple of pairwise commuting elements and that $\mathfrak{D}$ is an operating algebra. Then

$$
\operatorname{co} \sigma_{\mathfrak{D}}(\phi(x))=V_{\mathfrak{D}}(\phi(x))=V_{\mathfrak{R}}(x)=\sigma_{\mathfrak{B}}(x)=\operatorname{co} \sigma_{\mathfrak{R}}(x),
$$

where $\mathfrak{B}$ is an arbitrary $C^{*}$-subalgebra of $\mathfrak{R}$ containing $1_{\mathfrak{R}}, x_{1}, \ldots, x_{d}$. 


\section{The Weyl Functional CAlCulus}

Let $x=\left(x_{1}, \ldots, x_{d}\right) \in \mathfrak{R}^{d}$ be a hermitian element and let $E$ be the vector subspace of $\mathfrak{R}$ spanned by $1_{\mathfrak{R}}, x_{1}, \ldots, x_{d}$. Let $\phi: E \rightarrow \mathfrak{D}$ be a linear mapping with $\|\phi\|=1$ and $\phi\left(1_{\mathfrak{R}}\right)=1_{\mathfrak{D}}$. By Lemma 3.9] $\phi(x)$ is a hermitian element of $\mathfrak{D}$. Hence, for any function $f: \mathbb{R}^{d} \rightarrow \mathbb{C}$ with integrable Fourier transform $\hat{f}$, the following $\mathfrak{D}$ valued Bochner integral converges:

$$
f_{\mathfrak{R}}^{(\mathfrak{D}, \phi)}(x):=\int_{\mathbb{R}^{d}} \hat{f}(\lambda) e^{i \lambda \cdot \phi(x)} d \lambda .
$$

Here the Fourier transform $\hat{f}$ and the normalisation of Lebesgue measure $d x$ are chosen exactly so that when $\mathfrak{R}=\mathfrak{D}=\mathbb{C}$ and $\phi$ is the identity mapping $i d$, then $f(x):=f_{\mathbb{C}}^{(\mathbb{C}, i d)}(x)$ has the usual meaning.

Let $\mathcal{S}\left(\mathbb{R}^{d}\right)$ be the Schwartz space 8 of rapidly decreasing functions on $\mathbb{R}^{d}$. The mapping $W_{\mathfrak{R}}^{(\mathfrak{D}, \phi)}(x)$ given by $\left\langle W_{\mathfrak{R}}^{(\mathfrak{D}, \phi)}(x), f\right\rangle:=f_{\mathfrak{R}}^{(\mathfrak{D}, \phi)}(x)$ for all $f \in \mathcal{S}\left(\mathbb{R}^{d}\right)$ is continuous and linear on $\mathcal{S}\left(\mathbb{R}^{d}\right)$ and hence defines a tempered distribution taking values in the Banach algebra $\mathfrak{D}$.

Definition 4.1. The $\mathfrak{D}$-valued tempered distribution $W_{\mathfrak{R}}^{(\mathfrak{D}, \phi)}(x)$ is called the Weyl calculus of $x$ with respect to $\mathfrak{D}$ and $\phi$.

Let $i d$ be the identity mapping on $\mathfrak{R}$. We write $W_{\mathfrak{R}}(x)$ for $W_{\mathfrak{R}}^{(\mathfrak{R}, i d)}(x)$.

The following lemma is proved in [12] using the Trotter product formula for bounded self-adjoint operators on a Hilbert space. As every $C^{*}$-algebra is isometrically isomorphic to a closed subalgebra of bounded operators on some Hilbert space, we are able to state this lemma in slightly more generality.

Lemma 4.2 ([12], p.92). Suppose that $\mathfrak{D}$ is a unital $C^{*}$-algebra and let $x_{1}, x_{2}$ be hermitian elements of $\mathfrak{D}$. Then

$$
\left\|\exp \left(x_{1}+i x_{2}\right)\right\| \leq\left\|\exp x_{1}\right\| .
$$

For $z=\left(z_{1}, \ldots, z_{d}\right) \in \mathbb{C}^{d}$, let $\Im z:=\left(\Im z_{1}, \ldots, \Im z_{d}\right)$ and $\Re z:=\left(\Re z_{1}, \ldots, \Re z_{d}\right)$. The Hahn-Banach theorem ensures that there are sufficiently many linear functionals to obtain the following vector-valued extension of the usual Paley-Wiener theorem [8].

Theorem 4.3 (Paley-Wiener). An $\Re$-valued tempered distribution $u$ on $\mathbb{R}^{d}$ has compact support if and only if $u$ is the Fourier transform of an analytic function $e: \mathbb{C}^{d} \rightarrow \mathfrak{R}$ for which there exist constants $C \geq 0, s \geq 0$ such that $\|e(\zeta)\|_{\mathfrak{R}} \leq$ $C(1+|\zeta|)^{s} e^{r|\Im \zeta|}$ for all $\zeta \in \mathbb{C}^{d}$.

Theorem 4.4. Suppose that $\mathfrak{D}$ is a unital $C^{*}$-algebra. Then the distribution $W_{\mathfrak{R}}^{(\mathfrak{D}, \phi)}(x)$ has compact support.

Proof. We verify that the entire function $e(z):=\exp (i\langle z, \phi(x)\rangle)$, for $z \in \mathbb{C}^{d}$, satisfies the estimate required by Theorem 4.3 , We have

$$
\begin{aligned}
\|\exp i\langle z, \phi(x)\rangle\| & =\|\exp (\langle\Im z, \phi(x)\rangle-i\langle\Re z, \phi(x)\rangle)\| \\
& \leq\|\exp \langle\Im z, \phi(x)\rangle\| \\
& \leq \exp \|\langle\Im z, \phi(x)\rangle\| \\
& \leq \exp |\Im z|\|\phi(x)\| \\
& \leq \exp |\Im z|\|x\| .
\end{aligned}
$$


Let $C^{\infty}\left(\mathbb{R}^{d}\right)$ be the space of infinitely differentiable $\mathbb{C}$-valued functions on $\mathbb{R}^{d}$.

Corollary 4.5. Suppose that $\mathfrak{D}$ is a unital $C^{*}$-algebra. Then $W_{\mathfrak{R}}^{(\mathfrak{D}, \phi)}(x)$ extends uniquely to a distribution on $C^{\infty}\left(\mathbb{R}^{d}\right)$.

The following theorem gives a characterisation of the Weyl calculus distribution by its values on polynomials.

Theorem 4.6 ([2], Theorem 2.8). Suppose that $\mathfrak{R}$ is a unital $C^{*}$-algebra and let the monomial $p: \mathbb{R}^{d} \rightarrow \mathbb{R}$ be given by $p\left(\lambda_{1}, \ldots, \lambda_{d}\right):=\lambda_{1}^{k_{1}} \cdots \lambda_{d}^{k_{d}}$ for $k_{1}, \ldots, k_{d} \in \mathbb{N}$. Then $W_{\mathfrak{R}}(x)$ is the unique $\mathfrak{R}$-valued distribution such that

$$
\left\langle W_{\mathfrak{R}}(x), p\right\rangle=\frac{k_{1} ! \cdots k_{d} !}{k !} \sum_{\pi} x_{\pi(1)} \cdots x_{\pi(k)},
$$

where $k=k_{1}+\cdots+k_{d}$ and $\pi$ runs over the set of all maps from $\{1, \ldots, k\}$ into $\{1, \ldots, d\}$ that assume the value $j$ exactly $k_{j}$ times for $j=1, \ldots, d$.

Corollary 4.7. Suppose that $f \in C^{\infty}\left(\mathbb{R}^{d}\right)$ and for some $g \in C^{\infty}\left(\mathbb{R}^{d}\right), d^{\prime}<d$, $f\left(\xi_{1}, \ldots, \xi_{d}\right)=g\left(\xi_{1}, \ldots, \xi_{d^{\prime}}\right)$ for all $\xi_{1}, \ldots, \xi_{d} \in \mathbb{R}$. Then

$$
\left\langle W_{\mathfrak{R}}(x), f\right\rangle=\left\langle W_{\mathfrak{R}}\left(x^{\prime}\right), g\right\rangle,
$$

where $x^{\prime}=\left(x_{1}, \ldots, x_{d^{\prime}}\right)$.

If $\mathfrak{D}$ is an operating algebra, let

$$
I:=I(\mathfrak{D}, \phi)=\left\{y_{1} \in \mathfrak{D} \mid \operatorname{Sym}_{\mathfrak{R}}^{(\mathfrak{D}, \phi)}\left(y_{1} y_{2}\right)=0 \text { for all } y_{2} \in \mathfrak{D}\right\} .
$$

Then $I$ is a closed ideal of $\mathfrak{D}$, which allows us to define the quotient Banach algebra $\mathfrak{D}^{\prime}:=\mathfrak{D} / I$. Denote by $\phi^{\prime}$ the map induced by $\phi: E \rightarrow \mathfrak{D}$.

Lemma 4.8. Suppose that $\mathfrak{D}$ is an operating algebra with respect to $E$ and $\mathfrak{R}$. Then $\mathfrak{D}^{\prime}$ is also an operating algebra with respect to $E$ and $\mathfrak{R}$.

Proof. Clearly $\phi^{\prime}\left(1_{\mathfrak{R}}\right)=1_{\mathfrak{D}^{\prime}}$ and $\left\langle\phi^{\prime}(E)\right\rangle$ is dense in $\mathfrak{D}^{\prime}$. Since $S y m_{\mathfrak{R}}^{(\mathfrak{D}, \phi)}$ vanishes on $I$, the map $\operatorname{Sym}_{\mathfrak{R}}^{\left(\mathfrak{D}^{\prime}, \phi\right)}$ is well-defined and has all the properties required by Definition 2.1.

Theorem 4.9 ([1], Theorem 5.10; [11, Theorem 8). Suppose that $\mathfrak{D}$ is a unital commutative $C^{*}$-algebra and an operating algebra with respect to $E$ and $\Re$. Then

$$
\operatorname{supp} W_{\mathfrak{R}}^{\left(\mathfrak{D}^{\prime}, \phi^{\prime}\right)}(x)=\operatorname{supp} W_{\mathfrak{R}}(x)=\sigma_{\mathfrak{D}^{\prime}}\left(\phi^{\prime}(x)\right) .
$$

\section{A Canonical QUOtient operating Algebra}

Suppose that $V$ is a normed vector subspace of $\mathfrak{R}$ containing $1_{\mathfrak{R}}$. As in Section 2 , denote by $S(V)$ the symmetric algebra of $V$ and by $\bar{S}(V)$ the completion of $S(V)$ with respect to norm (2.3). Let ${ }^{\wedge}: V \rightarrow \bar{S}(V)$ be the canonical inclusion mapping.

Definition 5.1. Let $\mathcal{I}=\mathcal{I}(V):=\left\{\alpha \in \bar{S}(V) \mid \operatorname{Sym}_{\mathfrak{R}}^{(V)}(\alpha \beta)=0\right.$ for all $\left.\beta \in \bar{S}(V)\right\}$. The quotient $\mathcal{A}=\mathcal{A}(V):=\bar{S}(V) / \mathcal{I}$ is called the Nelson algebra of operants over $V$.

Let $^{\sim}: V \rightarrow \mathcal{A}$ be the canonical projection.

Lemma 5.2. $\tilde{1}_{\mathfrak{R}}=1_{\mathcal{A}}$. 
Proof. The unit in $\mathcal{A}$ is clearly the image of $1 \in \mathbb{C}$. However, for any $\alpha \in S(V)$,

$$
\begin{aligned}
\operatorname{Sym}_{\mathfrak{R}}^{(V)}\left(1-\hat{1}_{\mathfrak{R}}\right) \alpha & =\operatorname{Sym}_{\mathfrak{R}}^{(V)}\left(\alpha-\hat{1}_{\mathfrak{R}} \alpha\right) \\
& =\operatorname{Sym}_{\mathfrak{R}}^{(V)} \alpha-\operatorname{Sym}_{\mathfrak{R}}^{(V)} \hat{1}_{\mathfrak{R}} \alpha \\
& =0 .
\end{aligned}
$$

By continuity, $\operatorname{Sym}_{\mathfrak{R}}^{(V)}\left(1-\hat{1}_{\mathfrak{R}}\right) \alpha=0$ for all $\alpha \in \bar{S}(V)$. The result follows.

Lemma 5.3. The maps ${ }^{\wedge}: E \rightarrow S(E)$ and ${ }^{\sim}: E \rightarrow \mathcal{A}(E)$ are isometries. Furthermore, $\left\|^{\wedge}\right\|=\left\|^{\sim}\right\|=1$.

Theorem 5.4 ([11], Theorem 8). Suppose that $\mathfrak{R}$ is a unital $C^{*}$-algebra. Then

$$
\operatorname{supp} W_{\mathfrak{R}}^{\left(\mathcal{A}(E),{ }^{\sim}\right)}(x)=\operatorname{supp} W_{\mathfrak{R}}(x)=\sigma_{\mathcal{A}}(\tilde{x}) .
$$

Proof. By Lemma 2.2, $\bar{S}(E)$ is an operating algebra with respect to $E$ and $\mathfrak{R}$; hence by Lemma 4.8, $\mathcal{A}$ is an operating algebra with respect to $E$ and $\mathfrak{R}$. Since $\left\|^{\sim}\right\|=1$, the distribution $W_{\mathfrak{R}}^{\left(\mathcal{A}(E),{ }^{-}\right)}(x)$ is defined and equality (5.1) follows from Theorem 4.9 .

By Proposition 2.3 all operating algebras with respect to $E$ and $\mathfrak{R}$ are completions of quotients of $S(E)$ for some norm; hence $\mathcal{A}$ is the freest $(E, \mathfrak{R})$-operating algebra for which (4.1) holds.

Example 5.5. Let $A=\left(\begin{array}{cc}1 & 0 \\ 0 & -1\end{array}\right)$. Let $I$ be the identity of dimension 2 and set $E:=\operatorname{Span}_{\mathbb{C}}\{I, A\}$. Then $S(E) \cong \mathbb{C}[A]$. Since $A^{2}-I=0$ and $\operatorname{Sym}:=\operatorname{Sym}_{L\left(\mathbb{C}^{2}\right)}^{(E)}$ is a homomorphism we have $\left(\hat{A}^{2}-\hat{I}\right) \bar{S}(E) \subseteq \mathcal{I}(E)$. On the other hand, if $c_{0} \hat{I}+$ $c_{1} \hat{A}+\cdots+c_{n} \hat{A}^{n} \in \mathcal{I}(E)$, then

$$
\begin{aligned}
c_{0} I+c_{1} A+\cdots+c_{n} A^{n} & =\operatorname{Sym}\left(c_{0} \hat{I}+c_{1} \hat{A}+\cdots+c_{n} \hat{A}^{n}\right) \\
& =0
\end{aligned}
$$

and it follows that $\mathcal{I}(E)=(1-\hat{I}) \bar{S}(E)+\left(\hat{A}^{2}-\hat{I}\right) \bar{S}(E)$ by continuity.

Suppose that $\phi \in \sigma(\bar{S}(E))$. Then in particular $\phi(\mathcal{I}(E))=\{0\}$, or equivalently $\phi(1-\hat{I})=\phi\left(\hat{A}^{2}-I\right)=0$; hence $\phi(\hat{I})=1$ and $\phi(A)=\{-1,1\}$, and it follows that $\sigma(\hat{A})=\{-1,1\}$.

However, for $f \in \mathcal{S}(\mathbb{R})$ we have

$$
\begin{aligned}
\left\langle W_{L\left(\mathbb{C}^{2}\right)}(A), f\right\rangle & =\int_{\mathbb{R}} \hat{f}(\xi) e^{i \xi A} d \xi \\
& =\left(\begin{array}{cc}
f(1) & 0 \\
0 & f(-1)
\end{array}\right)
\end{aligned}
$$

and it follows that $\operatorname{supp} W_{L\left(\mathbb{C}^{2}\right)}(A)=\{-1,1\}$. Hence we have shown that $\sigma(A)=$ $\operatorname{supp} W_{L\left(\mathbb{C}^{2}\right)}(A)$.

\section{Spectra And matrix coefficients}

Let $G$ be a real, semisimple, compact and connected Lie group with Lie algebra $\mathfrak{g}$. Let $X:=\left(X_{1}, \ldots, X_{d}\right)$ be any basis of $\mathfrak{g}$ which is orthogonal with respect to the Killing form and such that for some $0<l \leq d, X^{\prime}:=\left(X_{1}, \ldots, X_{l}\right)$ is a basis of a Cartan subalgebra of $\mathfrak{g}$ which we fix and denote by $\mathfrak{t}$. Let $W$ be the Weyl group. Let $(\pi, \mathcal{H})$ be a unitary irreducible representation of $G$ of highest weight $\lambda$. We 
write $d \pi$ for the Lie derivative of $\pi$. In this section, we study the support of the Weyl calculus of $\frac{1}{i} d \pi(X):=\left(\frac{1}{i} d \pi\left(X_{1}\right), \ldots, \frac{1}{i} d \pi\left(X_{d}\right)\right)$.

Let $\mathfrak{g}^{*}$ be the dual of $\mathfrak{g}$. Let $\lambda_{1}, \ldots, \lambda_{n} \in \mathfrak{t}^{*}$ be the weights of $\pi$. Denote by $p: \mathfrak{g}^{*} \rightarrow \mathfrak{t}^{*}$ the canonical projection of $\mathfrak{g}^{*}$ onto $\mathfrak{t}^{*}$; i.e., $p(\xi):=\left.\xi\right|_{\mathfrak{t}^{*}}$ for all $\xi \in \mathfrak{g}^{*}$.

We write $W_{\pi}(X)$ for $W_{L(\mathcal{H})}\left(\frac{1}{i} d \pi(X)\right)$.

Definition 6.1. The moment set of $\pi$ is the set

$$
I_{\pi}:=\left\{\frac{1}{i}\langle d \pi(\cdot) u, u\rangle \mid u \in \mathcal{H},\|u\|=1\right\} .
$$

Theorem 6.2 ([3, 14]). Let $\pi$ be an irreducible representation of $G$ of highest weight $\lambda$. If the set $\{\lambda-w \lambda: w \in W\}$ does not contain a root, then $I_{\pi}=\operatorname{co} \mathcal{O}_{\lambda}$; otherwise, $I_{\pi}$ is not convex and $I_{\pi} \subsetneq \operatorname{co} \mathcal{O}_{\lambda}$.

Lemma 6.3. $p\left(I_{\pi}\right)=\operatorname{co}\left\{\lambda_{1}, \ldots, \lambda_{n}\right\}$.

Proposition 6.4. $\operatorname{cosupp} W_{\left.\pi\right|_{T}}\left(X^{\prime}\right)=\operatorname{co}(W \cdot \lambda)$.

Proof. Clearly $I_{\left.\pi\right|_{T}}=p\left(I_{\pi}\right)$. In view of Lemma 6.3, the result now follows from Theorem 3.11 and Theorem 3.2, and the fact that $\lambda_{1}, \ldots, \lambda_{n} \in \operatorname{co}(W \cdot \lambda)$.

Lemma 6.5. $\mathcal{O}_{\lambda} \subseteq I_{\pi}$.

Proof. We define the inclusion $\mathfrak{t}^{*} \subseteq \mathfrak{g}^{*}$ by the Killing form. Since $I_{\pi}$ is invariant under $A d^{*}$ and $\mathcal{O}_{\lambda} \cap \mathfrak{t}^{*}=W \cdot \lambda$, the statement follows from the containment $W \cdot \lambda \subseteq I_{\pi}$.

Proposition 6.6 ([3], Proposition 16). $G \cdot \operatorname{co}\left\{\lambda_{1}, \ldots, \lambda_{n}\right\}=\operatorname{co} \mathcal{O}_{\lambda}$.

By Lemma 3.3. the numerical range $V_{L(\mathcal{H})}\left(\frac{1}{i} d \pi(X)\right)$ does not depend on the choice of orthogonal basis $X$. We write $V_{\pi}$ for $V_{L(\mathcal{H})}\left(\frac{1}{i} d \pi(X)\right)$.

Theorem 6.7. $V_{\pi}=\operatorname{co} \mathcal{O}_{\lambda}$.

Proof. We have $I_{\pi}=G \cdot\left(I_{\pi} \cap \mathfrak{t}^{*}\right) \subseteq G \cdot p\left(I_{\pi}\right)=\operatorname{co} \mathcal{O}_{\lambda}$, where the last equality follows from Lemma 6.3 and Proposition 6.6] By Lemma 6.5 we have co $\mathcal{O}_{\lambda} \subseteq \operatorname{co} I_{\pi}$. Hence $\operatorname{co} I_{\pi}=\operatorname{co} \mathcal{O}_{\lambda}$, and the result follows from Theorem 3.2

Lemma 6.8. $\operatorname{supp} W_{\pi}(X)=G \cdot \operatorname{supp} W_{\pi}(X)$.

Proof. Suppose that $\xi \in \mathfrak{g}^{*} \backslash \operatorname{supp} W_{\pi}(X)$. Then there exists $\phi \in \mathcal{S}\left(\mathfrak{g}^{*}\right)$ such that $\phi$ is nonvanishing in a neighbourhood of $\xi$ and $\left\langle W_{\pi}(X), \phi\right\rangle=0$. Let $g \in G$. Set $\tilde{\phi}:=\phi \circ A d^{*}\left(g^{-1}\right)$. Then $\tilde{\phi} \in \mathcal{S}\left(\mathfrak{g}^{*}\right)$ and $\tilde{\phi}$ is nonzero near $g \cdot \xi$. Now,

$$
\begin{aligned}
\left\langle W_{\pi}(X), \tilde{\phi}\right\rangle & =\int_{\mathfrak{g}} \hat{\phi}(g \cdot X) e^{d \pi(X)} d X \\
& =\int_{\mathfrak{g}} \hat{\phi}(X) e^{d \pi\left(g^{-1} \cdot X\right)} d X \\
& =\pi\left(g^{-1}\right) \int_{\mathfrak{g}} \hat{\phi}(X) e^{d \pi(X)} d X \pi(g) \\
& =\pi\left(g^{-1}\right)\left\langle W_{\pi}(X), \phi\right\rangle \pi(g) \\
& =0 .
\end{aligned}
$$

Hence $g \cdot \xi \in \mathfrak{g}^{*} \backslash \operatorname{supp} W_{\pi}(X)$, and it follows that $G \cdot \operatorname{supp} W_{\pi}(X) \subseteq \operatorname{supp} W_{\pi}(X)$. The reverse inclusion is clear. This completes the proof. 
Theorem 6.9. $G \cdot \operatorname{supp} W_{\left.\pi\right|_{T}}\left(X^{\prime}\right) \subseteq \operatorname{supp} W_{\pi}(X)$.

Proof. By Corollary 4.7, supp $W_{\left.\pi\right|_{T}}\left(X^{\prime}\right) \subseteq \operatorname{supp} W_{\pi}(X)$, and the result follows by Lemma 6.8

Let $A$ be a subset of $\mathbb{R}^{d}$. The set of extremal points of $A$, denoted Ext $A$, is the collection of points in $A$ that are not contained in any open interval, the endpoints of which lie in $A$.

Lemma $6.10\left(\left[3\right.\right.$, Lemme 15). Ext $\operatorname{co}\left\{\lambda_{1} \ldots, \lambda_{n}\right\}=W \cdot \lambda$.

Corollary 6.11. $\operatorname{Ext} \operatorname{co} \mathcal{O}_{\lambda}=\mathcal{O}_{\lambda}$.

Theorem 6.12. $\operatorname{cosupp} W_{\pi}(X)=\operatorname{co} \mathcal{O}_{\lambda}$.

Proof. By Corollary 6.9,

$$
\begin{aligned}
\operatorname{cosupp} W_{\pi}(X) & \supseteq \operatorname{co} G \cdot \operatorname{supp} W_{\left.\pi\right|_{T}}\left(X^{\prime}\right) \\
& \supseteq G \cdot \operatorname{cosupp} W_{\left.\pi\right|_{T}}\left(X^{\prime}\right) \\
& =G \cdot V_{\left.\pi\right|_{T}},
\end{aligned}
$$

where the last equality follows from Theorem 3.11. By Theorem 6.7 $V_{\left.\pi\right|_{T}}=$ $\operatorname{co}\left\{\lambda_{1}, \ldots, \lambda_{n}\right\}$, and we have $\operatorname{cosupp} W_{\pi}(X) \supseteq \operatorname{co} \mathcal{O}_{\lambda}$ by Lemma 6.6. By Theorem 3.7. $\operatorname{cosupp} W_{\pi}(X) \subseteq V_{\pi}$ and the reverse inclusion follows. This completes the proof.

Theorem 6.13 ([8], Theorem 4.3.3). Let $u_{1}, u_{2}$ be compactly supported distributions on $\mathbb{R}^{d}$. Then

$$
\operatorname{cosupp} u_{1} * u_{2}=\operatorname{cosupp} u_{1}+\operatorname{cosupp} u_{2} \text {. }
$$

Lemma 6.14. $\operatorname{co} \mathcal{O}_{\lambda+\delta}=\operatorname{co} \mathcal{O}_{\lambda}+\operatorname{co} \mathcal{O}_{\delta}$.

Proof. We have

$$
\begin{aligned}
\operatorname{Ext}\left(\operatorname{co} \mathcal{O}_{\lambda}+\operatorname{co} O_{\delta}\right) & \subseteq \operatorname{Ext}\left(\operatorname{Extco} \mathcal{O}_{\lambda}+\operatorname{Extco} \mathcal{O}_{\delta}\right) \\
& =\operatorname{Ext}\left(\mathcal{O}_{\lambda}+\mathcal{O}_{\delta}\right) \\
& =\mathcal{O}_{\lambda+\delta}
\end{aligned}
$$

Therefore, by the Kreĭn-Milman theorem, $\operatorname{co} \mathcal{O}_{\lambda+\delta}$ is the closure of $\operatorname{co} \mathcal{O}_{\lambda}+\operatorname{co} \mathcal{O}_{\delta}$. Since the coadjoint orbits of $G$ are compact and the convex hull of a closed set is closed, the result follows.

Identifying the Lie algebra $\mathfrak{g}$ with $\mathbb{R}^{\operatorname{dim} G}$, let $\hat{j}$ denote the distributional Fourier transform of the $j$-function. It is well known that $\hat{j}=\mu_{\mathcal{O}_{\delta}}$ (see [4, Corollary 7.25).

Theorem 6.15. $\operatorname{cosupp} \hat{j} * W_{\pi}(X)=\operatorname{co} \mathcal{O}_{\lambda+\delta}$.

Proof. This is immediate from Theorem 6.13 and Theorem 6.12

Hence by Corollary 6.11, we reobtain the Kirillov-Duflo correspondence. 


\section{REFERENCES}

1. E. Albrecht, Several variable spectral theory in the noncommutative case, Spectral theory (Warsaw, 1977), Banach Center Publ., 8, 9-30, PWN, Warsaw, 1982. MR738273 (85h:47015)

2. R.F.V. Anderson, The Weyl functional calculus, J. Functional Analysis, 4 (1969), 240-267. MR 0635128(58:30405)

3. D. ARnal AND J. Ludwig, La convexité de l'application moment d'un groupe de Lie, J. Functional Analysis, 105 (1992), 256-300. MR1160080 (93j:22013)

4. N. Berline, E. Getzler and M. Vergne, Heat kernels and Dirac operators, Springer, Berlin, 2004. MR $2273508(2007 \mathrm{~m}: 58033)$

5. F.F. BONSALL AND J. DUNCAN, Numerical ranges of operators on normed spaces and of elements of normed algebras, Cambridge University Press, London, 1971. MR0288583 (44:5779)

6. A.H. Dooley, R.W. RAfFoul, Matrix coefficients and coadjoint orbits of compact Lie groups, Proc. Amer. Math. Soc., 135 (2007), 2567-2571. MR2302577 (2008d:43006)

7. D. Freed, M. Hopkins and C. Teleman, Loop groups and twisted K-theory. II, preprint.

8. L. HöRmANDER, The analysis of linear partial differential operators. I, Classics in Mathematics, Springer-Verlag, Berlin, 2003. MR.1996773

9. A.A. KiRILlov, Characters of unitary representations of Lie groups, Funkcional. Anal. i Priložen. 2 (1968), 40-55. MR0236318 (38:4615)

10. K.-H. NeEB, Holomorphy and convexity in Lie theory, Walter de Gruyter \& Co., Berlin, 2000. MR:1740617(2001j:32020)

11. E. Nelson, Operants: A functional calculus for non-commuting operators, Functional Analysis and Related Fields (Proc. Conf. for M. Stone, Univ. Chicago, Chicago, Ill., 1968), Springer, New York, 1970, pp. 172-187. MR0412857 (54:978)

12. M.E. TAYLOR, Functions of several self-adjoint operators, Proc. Amer. Math. Soc., 19 (1968), 91-98. MR 0220082 (36:3149)

13. M. Vergne, Representations of Lie groups and the orbit method, Emmy Noether in Bryn Mawr (Bryn Mawr, Pa., 1982), 59-101, Springer, New York, 1983. MR713793 (85a:22022)

14. N.J. Wildberger, The moment map of a Lie group representation, Trans. Amer. Math. Soc., 330 (1992), 257-268. MR.1040046 (92f:58064)

School of Mathematics and Statistics, University of New South Wales, Sydney, NSW 2052, Australia

E-mail address: raed@maths.unsw.edu.au 